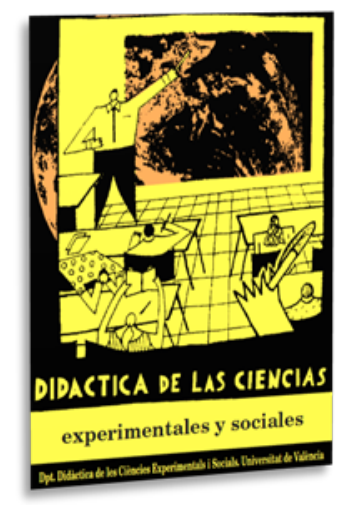

\title{
Alfabetización cartográfica: un desafío para la formación del profesorado
}

\author{
Cartographic Literacy: a challenge for teacher \\ training
}

DOI: $10.7203 / D C E S .40 .18054$

\author{
Francisco Rodríguez Lestegás \\ Universidad de Santiago de Compostela, f.lestegas@usc.es \\ ORCID iD: http://orcid.org/0000-0002-1733-3085 \\ Xosé Carlos Macía Arce \\ Universidad de Santiago de Compostela, carlos.macia@usc.es \\ ORCID iD: http://orcid.org/0000-0001-8597-4557 \\ Francisco Xosé Armas Quintá \\ Universidad de Santiago de Compostela, francisco.armas@usc.es \\ ORCID iD: http://orcid.org/0000-0002-8644-8185
}

\begin{abstract}
RESUMEN: El artículo defiende la relevancia de la geografía como saber estratégico y como forma de ver el mundo, lo que avala su utilidad como disciplina académica. Ahora bien, la enseñanza de la geografía debe entenderse en el sentido de una educación geográfica dirigida a promover la alfabetización geográfica, que se centra en la alfabetización espacial y en el desarrollo del pensamiento espacial omnipresente en nuestra vida diaria. La geografía contribuye a esta tarea a través de la alfabetización cartográfica, puesto que el mapa es un poderoso instrumento para alcanzar esta meta. No obstante, se revisa la deficiente utilización de los mapas en la escuela y se mencionan las limitaciones institucionales para asegurar una adecuada alfabetización cartográfica del profesorado. Finalmente, se afirma la necesidad de que los docentes reciban la formación suficiente en relación con las competencias necesarias para conducir el proceso de enseñanza y aprendizaje del lenguaje cartográfico.
\end{abstract}

PALABRAs CLAVE: geografía, mapas, profesorado, formación, enseñanza

ABSTRACT: This paper upholds the relevance of geography as strategic knowledge and as a way of looking at the world, which endorses its usefulness as scholar subject. However, the teaching of geography must be understood as geography education aimed at promoting geographic literacy, which is focused on spatial literacy and the development of spatial thinking that is omnipresent in our daily lives. Geography contributes to this task through cartographic literacy, since maps are powerful instruments to achieve this goal. However, the deficient use of maps in schools is reviewed and the institutional limitations to ensure adequate cartographic literacy among teachers are mentioned. Finally, the need for teachers to receive sufficient training in relation to the necessary competences to conduct the teaching and learning process of cartographic language is stated.

KEYWORDS: geography, maps, teachers, training, teaching.

Fecha de recepción: julio de 2020

Fecha de aceptación: noviembre de 2020

El artículo se enmarca en el Proyecto coordinado PGC2018-094491-B-C31: Las competencias docentes del profesorado para mejorar el aprendizaje de sus estudiantes (COMPROP). Subproyecto 1: Dimensión emocional y temas controvertidos en la formación del profesorado (EMOCOM). 


\section{INTRODUCCIÓN}

No es fácil encontrar respuestas satisfactorias en relación con la finalidad de la enseñanza de la geografía y la utilidad de su aprendizaje, y menos aún si se lo preguntamos a los estudiantes. Habitualmente, un buen alumno de geografía es el que presta atención en clase y luego aprende los conceptos y procesos geográficos gracias a un adecuado y esforzado trabajo personal. No es de extrañar, pues, que los escolares piensen que los contenidos geográficos que se ven obligados a estudiar tienen escasa aplicación en la realidad externa a la escuela (Cavalcanti, 1998, 2005).

En efecto, la geografía académica o escolar, lo que Lacoste (1977) denominaba la "geografía de los profesores", transmite la imagen de un saber enciclopédico, enumerativo e inocuo, sin aplicación práctica alguna al margen del propio sistema educativo que le da cobertura; en suma, una materia poco atractiva, que no despierta ningún interés entre los estudiantes (a veces tampoco entre el profesorado encargado de impartirla) y en la que basta con memorizar unos contenidos normalmente aburridos para salir airoso de las pruebas de evaluación.

Hay otra geografía igualmente de escasa relevancia, la "geografía espectáculo" (Lacoste, 1977), que es la que practican las oficinas de turismo, las agencias de viajes, los medios de comunicación o la publicidad. Este relato geográfico banaliza y mercantiliza los paisajes para su consumo masivo, nos sitúa ante ellos con una actitud de contemplación pasiva y de simple admiración estética, a la vez que desactiva la comprensión crítica de las estrechas relaciones entre saber geográfico, ideología y poder.

Ahora bien, frente a la irrelevancia de la "geografía de los profesores" y de la "geografía espectáculo", Lacoste destaca la consideración de la geografía como saber estratégico que las minorías dirigentes utilizan como instrumento de poder. Estrechamente unida a las prácticas políticas y militares, la geografía "sirve, en primer lugar, para hacer la guerra y ejercer el poder" (Lacoste, 1977, p. 8).

Más recientemente, Gersmehl (2014) añadió que la geografía, además de ofrecer un cuerpo de conocimientos, es singularmente una forma de ver el mundo que guarda una estrecha relación con el alcance estratégico que le procura relevancia. En defensa de esta consideración, Gersmehl propone la utilización de una perspectiva geográfica para comparar la situación de Egipto e Irak ante la oleada revolucionaria iniciada en 2011 y conocida como "Primavera Árabe". Con el fin de proporcionar una explicación verosímil a las radicalmente diferentes decisiones políticas y militares adoptadas en cada uno de esos dos países, se fija en un único factor: los ingresos que ambos gobiernos obtenían de la venta del petróleo y que utilizaban, aparte de la compra de armamento, para financiar ciertos servicios o ayudas sociales, tales como educación gratuita o alimentos subvencionados. Cuando Gersmehl compara las tendencias de los ingresos por la venta de crudo entre 1970 y 2010, observa un comportamiento claramente distinto entre los dos países, lo que explicaría la caída del régimen del dictador egipcio Hosni Mubarak sin necesidad de ninguna intervención extranjera y, en cambio, la terrible guerra de Irak como única fórmula que las grandes potencias occidentales encontraron para acabar con el régimen de Saddam Hussein. Esta argumentación es lo que conduce a Gersmehl a concluir que la perspectiva geográfica nos ayuda a entender por qué algo que se cumple en un lugar puede no hacerlo en otro, incluso teniendo características espaciales aparentemente similares, de manera que el conocimiento aportado por un enfoque geográfico nos facilita la comprensión de la actividad y del comportamiento que los grupos humanos desarrollan en un territorio concreto.

\section{ALFABETIZACIÓN GEOGRÁFICA Y ALFABETIZACIÓN ESPACIAL}

La consideración de la geografía como saber estratégico y como conocimiento que permite la adopción de una perspectiva idónea para la comprensión de nuestro mundo no deja lugar a dudas sobre la utilidad de esta disciplina académica. El problema está en que muy pocas veces la 
geografía escolar ofrece realmente estas posibilidades. Por si fuera poco, el alumnado tampoco le reconoce a la geografía ninguna influencia sobre su formación como ciudadanos, pese a que la Declaración Internacional sobre la Educación Geográfica, promulgada en Pekín en 2016, subraya que los conocimientos geográficos son indispensables para la conformación de una ciudadanía responsable y activa.

Definitivamente, los estudiantes tienen bastante claro que la geografía que aprenden conjuga la utilidad cultural con la inutilidad formativa (Audigier, 1994), motivo por el que corre el serio riesgo de convertirse en una materia innecesaria, superflua, decorativa y, por lo tanto, perfectamente prescindible. Ante estos síntomas, parece que el diagnóstico no es difícil: el menosprecio de la utilidad de la geografía como disciplina escolar es una lógica consecuencia de la desconexión entre enseñanza de la geografía y educación geográfica.

La principal tarea de la educación geográfica es promover la "alfabetización geográfica", cuyo componente de mayor rango es la "alfabetización espacial”. Se trata de un concepto relativamente nuevo que incluye tanto conocimientos como capacidades de actuación en el espacio, es decir, competencias espaciales. En realidad, la alfabetización espacial no es algo que deba adquirirse separadamente de otros aprendizajes escolares (lingüísticos, matemáticos, científicos, artísticos, motrices), sino que todos ellos están estrechamente relacionados y forman parte del mismo proceso de aprender a pensar (Sinton, Bednarz, Gersmehl, Kolvoord y Uttal, 2013).

En este sentido, podemos decir que una persona está alfabetizada espacialmente cuando es capaz de "pensar el espacio" de una manera informada, reflexiva y crítica, utilizando sus concepciones y representaciones espaciales para resolver adecuadamente las tareas y problemas que se le presentan en su vida diaria.

\subsection{El pensamiento espacial: pensar “en”, “sobre” y “con” el espacio}

El desarrollo del pensamiento espacial es el objetivo primordial de la alfabetización espacial. Se trata de un proceso cognitivo complejo basado en una combinación constructiva de tres elementos: conceptos espaciales (localización, posición, dirección, movimiento, distancia, orientación), instrumentos de representación espacial (un mapa, pero también un modelo atómico o el diagrama tridimensional de la estructura de una molécula) y procesos de razonamiento espacial (relacionados con la percepción, la rotación mental y la visualización). En este sentido, el pensamiento y razonamiento espacial surge de un aprendizaje basado, entre otros elementos, en el uso de conceptos espaciales y herramientas para el conocimiento territorial que permitan aplicar la información espacial obtenida a la resolución de problemas del mundo real. No obstante, debemos ser conscientes de que el pensamiento espacial está insuficientemente reconocido, escasamente valorado y, por lo tanto, cuenta con una débil presencia en los currículos escolares (National Research Council, 2006; Sinton et al., 2013; Zwartjes y de Lázaro, 2019).

Con todo, el pensamiento espacial está activo en todas las facetas de nuestra vida. Lo está, en primer lugar, en nuestros espacios cotidianos, que es cuando pensamos "en” el espacio. Por ejemplo, cuando ajustamos los espejos retrovisores del automóvil, cuando entramos en una estación de servicio y situamos el coche del lado correcto junto al surtidor para repostar combustible, cuando tratamos de aparcarlo, cuando pensamos cómo bajar un piano por unas escaleras, cuando ordenamos un armario o una estantería, cuando tratamos de sacar el máximo provecho a la capacidad de un lavavajillas, cuando preparamos nuestra maleta para realizar un viaje, cuando intentamos acomodar nuestro equipaje de mano en el compartimento superior de un avión, cuando una empresa de catering organiza las bandejas de comida para esa misma aeronave, cuando explicamos a alguien como llegar a un lugar en una ciudad que desconoce, o cuando mantenemos la distancia de seguridad entre vehículos (Duarte, 2017; Sinton et al., 2013).

Existen otras circunstancias en las que pensamos "sobre" el espacio. Nos referimos al tipo de pensamiento que corresponde a los espacios físicos y sociales, en donde vivimos y por donde nos 
desplazamos diariamente. Es el espacio que condiciona nuestras vidas por sus rasgos climáticos, por las crecidas de los ríos, por las variaciones de las mareas, por las características de los suelos o por los riesgos geológicos. Así, pensamos sobre el espacio físico cuando conducimos un vehículo y tenemos que calcular el momento en que debemos empezar a frenar para que se detenga justo en el lugar apropiado, teniendo en cuenta la variación de la distancia de frenada en función de las condiciones del asfalto (seco, mojado, nevado). Igualmente, es necesario prestar atención a las condiciones físicas del espacio cuando se diseñan uniformes militares con la intención de facilitar el camuflaje en el desierto o en la selva. El pensamiento sobre los espacios físicos también es el que tienen que desarrollar los urbanistas o planificadores urbanos, los ingenieros que diseñan el trazado de las vías de comunicación y otras infraestructuras, y los arquitectos que se responsabilizan de proyectos constructivos. Por otro lado, nos situamos en el dominio geográfico de los espacios sociales cuando elegimos un determinado restaurante, bar, cafetería o discoteca para disfrutar de nuestros momentos de ocio, o cuando decidimos vivir en un determinado barrio de la ciudad. El pensamiento sobre los espacios sociales nos sirve también para analizar los espacios de exclusión derivados de los procesos de segregación socioespacial por motivos económicos, de raza, género o cualquier otra condición (Duarte, 2017; Sinton et al., 2013).

Finalmente, pensamos “con” el espacio cuando apelamos a los espacios intelectuales, es decir, los que se utilizan para facilitar la comprensión de informaciones abstractas y la organización del conocimiento, así como la transmisión de su significado. Por ejemplo, jugar al ajedrez supone el desarrollo de una estrategia espacial que tiene por objetivo atacar al rey del oponente de modo que se imposibilite totalmente su defensa. Así mismo, los mapas conceptuales presentan los conceptos ordenados jerárquicamente por orden de importancia o inclusión, situándolos en la estructura gráfica siguiendo una secuencia espacial desde la parte superior a la inferior. La organización espacial también está presente en los árboles genealógicos, representación gráfica que muestra de forma sistemática las relaciones parentales que unen a los miembros de una familia. Los organigramas de empresas o instituciones también muestran en un formato espacial su estructura departamental, así como las relaciones jerárquicas y competenciales entre las personas que las dirigen. La tabla periódica de los elementos químicos representa otro buen ejemplo de herramienta gráfica que obliga a pensar con el espacio, ya que los elementos que contiene se encuentran ordenados por filas en orden creciente de su número atómico, al tiempo que este ordenamiento muestra tendencias periódicas que permiten agrupar en columnas los grupos de elementos con propiedades químicas parecidas. Para terminar, acudimos a la geografía de nuestros espacios intelectuales cuando nos sorprendemos ante el modelo de doble hélice del ADN, que esquematiza la estructura molecular del ácido desoxirribonucleico (Duarte, 2017; Sinton et al., 2013).

\subsection{Pensar el espacio: la alfabetización cartográfica}

Junto con otras disciplinas escolares que asumen la responsabilidad de contribuir al desarrollo del pensamiento o razonamiento espacial, la geografía lo hace singularmente a través de la llamada “alfabetización cartográfica” (Cavalcanti, 2012, p. 25; Duarte, 2017, p. 32; Luque, 2011, p. 185), un proceso formativo que requiere el empleo de una metodología específica orientada al desarrollo de las estructuras cognitivas y habilidades que permiten interpretar y comprender el lenguaje cartográfico, así como construir significados a partir del mismo (Jerez, 2006).

Señalemos, en primer lugar, que no es posible entender la geografía sin cartografía y, además, perdería toda su identidad como ciencia. El mapa es una herramienta fundamental en la explicación geográfica y, por lo tanto, un instrumento básico para el trabajo de los geógrafos, tal como dejó señalado Hartshorne (1939) hace varias décadas. En el mismo sentido, Yves Lacoste se refería al mapa como "la forma de representación geográfica por excelencia" (Lacoste, 1977, p. 7). Y es que, en realidad, estamos hablando de un instrumento imprescindible para la comprensión de los 
fenómenos espaciales, para almacenar la información eficientemente y para entender las distribuciones y relaciones geográficas (Thrower, 2002).

En el plano educativo, tampoco es posible enseñar ni aprender la geografía sin mapas, ya que supondría abordar un proceso didáctico totalmente al margen de las realidades espaciales. Sin ningún género de dudas, el alumnado se encontraría con innumerables dificultades para asociar la realidad física, humana y económica con territorios concretos. Por eso, además de seña distintiva del geógrafo, el mapa es "el documento básico de gran parte de la enseñanza de la geografía" (Bailey, 1981, p. 36). Ahora bien, "leer” el mapa, es decir, comprender, interpretar y utilizar, no solo toda la información que proporciona, sino también la que ignora, oculta o distorsiona, exige un complejo proceso de enseñanza y aprendizaje (Thrower, 2002).

\subsection{El mapa como construcción social etnocéntrica}

Los mapas no solo nos permiten localizar puntos en el espacio, orientarnos, comparar dimensiones y otras actividades de carácter matemático que el enfoque neopositivista se ha encargado de priorizar, sino que también nos ofrecen la posibilidad de transmitir una determinada visión o interpretación sobre la organización del espacio humanizado. En este sentido, lejos de proporcionar una imagen fiel, objetiva y neutral de la realidad espacial, los mapas son construcciones sociales que transmiten mensajes ideológicos, culturales y políticos.

Dibujar un mapa no es, pues, una operación tan aséptica como pudiera parecer. Por el contrario, la intencionalidad del lenguaje cartográfico adquiere un interés prioritario, ya que cada mapa pretende dar respuesta a un asunto concreto, lo que representa una determinada selección y presentación de la información para lograr una visualización eficaz de la cuestión que lo ha justificado. El proceso de adquisición de las habilidades cartográficas no debe quedar, pues, reducido a una simple familiarización con el lenguaje convencional que presentan los mapas, sino que debe enfocarse hacia la comprensión del sistema de comunicación gráfica (Benejam y Comes, 1994).

Para empezar, tenemos que asumir la certeza de que los mapas siempre han ofrecido una visión etnocéntrica del mundo. En la época de las cruzadas, desarrolladas entre 1096 y 1291, el gran objetivo de la cristiandad era la liberación de los Santos Lugares de la dominación musulmana, por lo que la ciudad de Jerusalén se convirtió en el lugar de culto por excelencia y ocupaba, por ello, el centro de los mapas producidos por los cartógrafos cristianos, que todavía representaban la Tierra como un disco plano de acuerdo con las enseñanzas de Isidoro de Sevilla. De manera análoga, la cartografía árabe, entonces muy superior técnicamente a la europea, situaba la ciudad santa musulmana de La Meca en el centro de sus planisferios. Así, tanto en Occidente como en Oriente se impuso un criterio cartográfico de inspiración religiosa según el cual los lugares de culto ocupaban el centro de los mapas. Los mapas medievales eran, de este modo, tan bellos como erróneos y falsos, porque su propósito no era representar la realidad espacial, sino ofrecer un producto iconográfico que proporcionase una determinada imagen etnocéntrica del mundo (Peters, 1992).

Pero las visiones etnocéntricas configuradas a través de los mapas perduran en la actualidad. Si le preguntamos a alguien de nuestro entorno cómo es el mundo, su imagen mental probablemente no será muy distinta de esta: América a la izquierda, Europa en el centro y Asia a la derecha. Esto es debido a que los mapas europeos occidentales han tendido a representar el mundo con su eje oeste-este centrado sobre Europa Occidental y el meridiano de Greenwich, adoptado en 1884 como meridiano $0^{\circ}$. La visión estadounidense del mundo, como es de esperar, sitúa el eje oeste-este centrado sobre Estados Unidos, lo que provoca que Eurasia quede dividida por la mitad y que la India aparezca dos veces en el mapa. Por su parte, el mapamundi de China centra el mundo en el Océano Pacífico, con el fin de situar el gigantesco país asiático en medio del eje oeste-este. Finalmente, la visión desde Australia ofrece una perspectiva del mundo de abajo a arriba o, lo que es lo mismo, el mundo al revés; es una forma de mostrar que la orientación convencional, con el 
norte en la parte superior del mapa, es totalmente arbitraria y que podría estar en cualquier otra posición: "norte” y "arriba” no son sinónimos, de igual manera que tampoco lo son "este” y “derecha”.

Como puede observarse, detrás de la elección de una mirada para el mapamundi siempre hay unas connotaciones relacionadas con el ejercicio del poder, sea este del signo que sea. La superficie esférica terrestre no tiene un centro, pero su proyección sobre una superficie plana supone la elección de un centro y de unos bordes o límites, algo nada ingenuo y de persistentes consecuencias (Grataloup y Fumey, dirs., 2016). La elección de una u otra perspectiva marca la visión que sucesivas generaciones, nacidas y educadas en un determinado territorio, tienen del planeta. Una cuestión decisiva que nace a partir de los mapas.

\subsection{Elegir una u otra proyección cartográfica no es indiferente}

No es posible una representación fidedigna de la superficie terrestre en dos dimensiones. Pasar de una superficie curva a otra plana viene a ser una especie de "domesticación del espacio" que supone elegir necesariamente entre dos cualidades excluyentes entre sí: o bien se respetan las formas conservando los ángulos, pero deformando las superficies (proyección conforme), o bien se respetan las superficies, pero deformando los perfiles de los continentes (proyección equivalente) (Grataloup y Fumey, dirs., 2016).

En este sentido, es sabido que los mapamundis suelen estar confeccionados a partir de la proyección ideada en 1569 por Gerardus Mercator, que sirvió para configurar una imagen gráfica y mental del mundo que perduraría hasta la actualidad. El cartógrafo flamenco utilizó en sus mapas una proyección cilíndrica conforme que distorsiona claramente las superficies, por lo que, siendo muy apropiada para la navegación, resulta en cambio poco adecuada para representar las distribuciones geográficas sobre la Tierra (Thrower, 2002). Dado que todos los meridianos y paralelos se cruzan en ángulo recto, los polos se desplazan hacia el infinito. Además, el ecuador no divide el planeta en dos mitades iguales, sino que las dos terceras partes del mapa se emplean para representar el hemisferio norte mientras que se dedica solo el tercio restante al hemisferio sur, de manera que los países norteños ven reforzada su preeminencia sobre los meridionales (Peters, 1992). Las distorsiones de superficie favorecen a los territorios situados en la zona templada del hemisferio norte, donde se ubicaban las potencias coloniales habitadas por poblaciones blancas, motivo por el cual, además de eurocentrista, el mapa de Mercator fue acusado de colonialista e, incluso, de racista.

Por el contrario, el alemán Arno Peters creó en 1974 un mapamundi basado en una proyección equivalente que conserva las superficies, aunque deforma los perfiles de los continentes. Peters considera que la cualidad más importante de un mapa es la fidelidad de superficie, porque facilita las comparaciones entre países, continentes y océanos, al tiempo que otorga el mismo rango a todos los pueblos (Peters, 1992). Para ello, sitúa el ecuador dividiendo el planisferio por la mitad y consigue que un centímetro cuadrado en cualquier punto del mapa represente los mismos quilómetros cuadrados en la realidad. Al tratar de representar un mundo poscolonial con una mayor equidad, esta proyección fue adoptada por numerosas organizaciones y utilizada en materiales educativos, pero no pudo librarse de ser considerada un producto absurdo, provocador y falsificador.

En todo caso, el uso de una u otra proyección no resulta indiferente. Pensemos, por ejemplo, en un mapa que tenga por objeto la representación de la desigualdad de la distribución de la riqueza en el mundo. La cartografía realizada con la proyección Mercator escamotea gran parte de la gravedad del problema, mientras que si se utiliza el mapa de proyección Peters los desequilibrios se nos presentan con mayor rotundidad. Y lo mismo podría decirse respecto a la esperanza de vida, mortalidad infantil, consumo de calorías, población que vive en ciudades, promedio de ingresos por 
persona, trabajo infantil, analfabetismo o cualquier otro indicador que exprese las desigualdades sociales en nuestro planeta (Calaf, Suárez y Menéndez, 1997).

En definitiva, es evidente que tanto las personas como las organizaciones siempre han usado los planisferios para sus propios fines simbólicos, ideológicos y políticos, al margen de los criterios técnicos de exactitud que persiguen los cartógrafos. Los mapas siempre son representaciones selectivas y parciales del territorio, por lo que su utilización no puede escapar de los prejuicios personales ni de la manipulación política (Brotton, 2014).

\subsection{La distorsión deliberada de los mapas}

En ocasiones, es preciso que los mapas representen de forma muy gráfica una visión del espacio que facilite la comprensión de determinados problemas y conflictos territoriales, lo que obliga a deformar intencionadamente las superficies para proporcionar una imagen fuertemente expresiva de la realidad. Por ejemplo, en un cartograma de la población mundial que muestre los tamaños de los países en proporción a su volumen demográfico, Australia y Canadá estarán casi ausentes, en tanto que la India aparecerá cartografiada con un tamaño superior al de África.

De manera semejante, si se ajustan los tamaños de los países en relación directa a las tasas de población encarcelada, destaca la enorme dimensión que alcanza este problema en Estados Unidos. En todo caso, hay que señalar dos certezas: por un lado, no existe una correlación directa entre los índices de encarcelamiento y de criminalidad; por otro, las minorías étnicas y sociales están sobreencarceladas (Grataloup y Fumey, dirs., 2016).

Y en un mapa distorsionado de acuerdo con la riqueza de los países, el sur prácticamente no existe.

\section{Pensamiento espacial Y AlFABetización CARTográfica EN EL CURRÍCULO Y EN LOS LIBROS DE TEXTO}

Como hemos visto, una dimensión clave de la educación geográfica es el desarrollo del razonamiento espacial, consistente en la progresión de la capacidad para visualizar e interpretar la localización, la posición, la dirección, el movimiento, la distancia y la orientación sobre el espacio. Se trata de una faceta del pensamiento que se utiliza en todo tipo de situaciones, a muy diversas escalas y que se manifiesta a través de distintas acciones y estrategias. Por esta razón, las personas poseen diferentes competencias espaciales y, en función de ellas, destacarán en unos u otros tipos de pensamiento espacial, que se ve notablemente afectado por los conocimientos previos y las experiencias desarrolladas en el entorno vital de cada individuo.

En el ámbito escolar, los alumnos tienen que utilizar el pensamiento espacial en numerosas ocasiones: cuando realizan construcciones con bloques, cuando señalan la dirección que tienen que seguir para ir desde su casa hasta la escuela u otros emplazamientos importantes de la localidad, cuando adquieren conocimientos escolares (no solo geográficos) de diversa índole, etc. El progreso académico implica necesariamente la utilización de una creciente gama de competencias espaciales, pero a pesar de ello el pensamiento espacial suele estar ausente de la relación de objetivos curriculares y de resultados de aprendizaje (Sinton et al., 2013).

Creemos haber dejado claro que el mapa representa un poderoso aliado para alcanzar esta meta, ya que el lenguaje cartográfico es el código idóneo para la transmisión de la información geográfica. Sin embargo, el currículo vigente (Tabla 1) propicia que el aprovechamiento didáctico de los mapas se limite a su papel como soporte de localizaciones que sirven para verificar el discurso enunciado por el profesor o escrito en el libro de texto que manejan los alumnos y que, en definitiva, contiene la información que hay que aprender (Fontanabona, dir., 2000), constituida en la mayor parte de las ocasiones por enunciados obvios y explicaciones convencionales. 
TABLA 1. Prescripciones curriculares para el empleo del mapa en la escuela

\begin{tabular}{|c|c|}
\hline \multicolumn{1}{|c|}{ ESTÁNDARES DE APRENDIZAJE EVALUABLES } \\
\hline Educación Primaria: Ciencias Sociales \\
- $\quad$ Localizar: diferentes puntos de la Tierra empleando los paralelos y meridianos y las \\
coordenadas geográficas; las principales unidades de relieve de España y sus vertientes \\
hidrográficas; el relieve de Europa, sus vertientes hidrográficas y sus climas. \\
- $\quad$ Situar: los mares, océanos y los grandes ríos de España; los mayores núcleos de \\
población en España y las zonas más densamente pobladas. \\
- $\quad$ Señalar: los tipos de climas de España y las zonas a las que afecta cada uno. \\
\hline Educación Secundaria Obligatoria: Geografía e Historia \\
- Localizar: un punto geográfico en un planisferio; espacios geográficos y lugares en un \\
mapa utilizando datos de coordenadas geográficas; los grandes conjuntos o espacios \\
bioclimáticos de España; las principales unidades y elementos del relieve europeo; los \\
distintos tipos de clima de Europa; las zonas bioclimáticas de nuestro continente; los \\
principales elementos y referencias físicas: mares y océanos, continentes, islas y \\
archipiélagos más importantes, además de los ríos y las principales cadenas \\
montañosas; los continentes y las áreas más densamente pobladas; las principales \\
zonas productoras de minerales en el mundo; las principales zonas productoras y \\
consumidoras de energía en el mundo; los países más industrializados del mundo. \\
Situar: las principales unidades de relieve español, europeo y mundial; las veinte \\
ciudades más pobladas; las principales zonas cerealícolas y las más importantes masas \\
boscosas del mundo.
\end{tabular}

Fuente: Real Decreto 126/2014, de 28 de febrero, por el que se establece el currículo básico de la Educación Primaria (BOE del 1 de marzo de 2014) y Real Decreto 1105/2014, de 26 de diciembre, por el que se establece el currículo básico de la Educación Secundaria Obligatoria y Bachillerato (BOE del 3 de enero de 2015).

Muchos de los mapas topográficos y temáticos incluidos en los libros de texto (currrículo presentado) cumplen una función meramente ilustrativa, lo que les vale ser sistemáticamente ignorados tanto por el profesorado como por los estudiantes, o bien se limitan a completar la información escrita que proporciona el manual. Se trata, en su mayoría, de mapas coropléticos o corocromáticos, poco indicados para la puesta en práctica de una metodología activa, reflexiva y crítica. En cambio, los materiales relacionados con la cartografía digital y la geoinformación tienen una escasa presencia en los libros de texto, cuando se trata de recursos didácticos esenciales para el desarrollo del pensamiento espacial y la implementación de propuestas innovadoras en la enseñanza de la geografía (De Miguel, 2013).

En este escenario, las actividades de aprendizaje propuestas para trabajar con documentos cartográficos no requieren, en la mayoría de las ocasiones, observar el mapa en cuestión: basta con recoger la información solicitada directamente del texto que lo acompaña o, como mucho, efectuar alguna comprobación en relación con su localización cartográfica. A lo que habría que añadir las numerosas actividades que remiten a la utilización de mapas mudos con la única finalidad de localizar y nombrar determinados puntos geográficos, ya sean países, ciudades, montañas, ríos o cualquier otro emplazamiento mencionado en la correspondiente lección. De este modo, el mapa no se "lee" en la escuela; no se extrae la información que contiene, ni se analiza, ni se interpreta, lo cual, por otra parte, solo sería posible trabajando con mapas dibujados a escalas muy superiores a las que aparecen normalmente en los mapas escolares (Catalá, 2016; Richter y Cavallini, 2019).

Esto hace que los mapas en la escuela se utilicen a menudo para la realización de actividades que priorizan la localización y la descripción sobre cualquier otro procedimiento, renunciando a su potencialidad para aprender a pensar el espacio y adquirir el dominio del razonamiento geográfico, las dos orientaciones esenciales de la didáctica de nuestra disciplina. La cartografía escolar se desentiende así de procesos intelectuales relevantes y pasa a formar parte del "currículo nulo", es decir, contenidos que están ausentes del currículo explícito; lo que debería ser ofrecido y no lo es; 
lo que la escuela omite y no enseña, pero que es tanto o más importante que aquello que sí enseña (Eisner, 1979). Al mismo tiempo, la elección de las proyecciones en un mapa constituye un buen ejemplo de "currículo oculto", entendiendo por tal todos aquellos aspectos del ambiente escolar que, sin formar parte del currículo explícito, contribuyen implícitamente a los aprendizajes sociales relevantes (Jackson, 1968).

\section{ALFABETIZACIÓN CARTOGRÁFICA Y FORMACIÓN DEL PROFESORADO}

La alfabetización cartográfica es un componente de la alfabetización gráfica (lo que en el ámbito anglosajón se denomina "graphicacy"), que debemos entender como la capacidad de comprender y presentar información en forma de gráficos, diagramas, imágenes, fotografías, croquis, planos, mapas y otros formatos no textuales (Sinton et al., 2013). No obstante, siendo el mapa el lenguaje específico y la principal opción metodológica de la geografía, paradójicamente no es el medio más utilizado para la transmisión y recepción de la información en el proceso de enseñanza-aprendizaje de esta disciplina. La razón es muy sencilla: lo que ocurre, simplemente, es que el profesorado encargado de gestionar este proceso no ha recibido la formación adecuada para utilizar la cartografía como instrumento de comunicación, limitándose a utilizar el mapa como instrumento de apoyo docente o material complementario para la realización de actividades por parte de los escolares. Por consiguiente, buena parte del alumnado desconoce las claves necesarias para extraer e interpretar adecuadamente toda la información que los mapas contienen, tales como los códigos propios del lenguaje cartográfico (simbología, iconografía, colores, tramas) o la función de la escala (Catalá, 2016).

Quienes aspiran a ejercer como maestros de educación primaria acreditan escasas habilidades cartográficas y, en ocasiones, incluso poco interés en adquirirlas. Una proporción no despreciable de los estudiantes del grado universitario correspondiente tiene un escaso conocimiento de los aspectos matemáticos propios del lenguaje cartográfico (proyecciones, coordenadas geográficas, orientación, escala) y tampoco posee las claves necesarias para reflexionar sobre los mensajes ideológicos, culturales y políticos que transmiten los mapas, a través de los que se propaga una determinada visión o interpretación sobre la organización del espacio humanizado.

La situación no es mucho más halagüeña en el caso de los futuros docentes de geografía en la educación secundaria. Es sabido que solo una mínima parte del alumnado que cursa la titulación de máster que habilita para el desempeño profesional en ese nivel, con la expectativa de acceder a la especialidad docente de Geografía e Historia, ha seguido previamente los estudios del grado en Geografía, siendo la mayoría graduados en Historia, Historia del Arte u otras titulaciones de lo más diverso y, por lo tanto, con escasa o nula formación cartográfica. En este sentido, no es de extrañar que los docentes de Educación Secundaria acusen un desconocimiento generalizado de los sistemas de información geográfica, lo que se traduce en una desconsideración de la enseñanza de fuentes de información digitales propias de la geografía, junto a la renuncia a utilizar recursos y materiales didácticos de carácter virtual (Catalá, 2016; Nieto y Siegmund, 2019).

Por otro lado, es sabido que los manuales escolares son en la mayoría de los casos la principal o incluso única guía de la práctica docente. Pues bien, ya ha quedado señalado que los libros de texto utilizan la cartografía para reforzar la visión de la geografía como una disciplina académica, descriptiva y memorística, retrasando su evolución hacia una ciencia explicativa del mundo actual y de los procesos sociales que tienen lugar en el territorio y contribuyendo a que el proceso de enseñanza-aprendizaje de esta materia se aleje significativamente de las propuestas innovadoras que ya se abren paso en otros países de nuestro entorno (De Miguel, 2013).

Así, pues, parece sobradamente justificado el papel de la alfabetización cartográfica en los planes de estudio de las titulaciones de grado y máster habilitantes para el ejercicio de las profesiones reguladas de maestro y profesor de educación secundaria. Sin embargo, las órdenes ministeriales por las que se establecen los requisitos para la verificación de los títulos universitarios 
oficiales de grado y máster correspondientes (Orden ECI/3857/2007 y Orden ECI/3858/2007, respectivamente) no dejan mucho margen de maniobra: las directrices reservan 100 créditos para el módulo didáctico y disciplinar en la formación de maestros de educación primaria, que está dirigido a la adquisición de las competencias propias de siete áreas de conocimiento; por su parte, el módulo específico del máster en profesorado de educación secundaria cuenta con solo 24 créditos para su desarrollo.

Las necesidades formativas del profesorado son especialmente notorias cuando nos referimos a la utilización de la cartografía digital y los sistemas de información geográfica (SIG) como recurso educativo. En efecto, la revolución de las tecnologías de la información y de la comunicación, que tuvo sus inicios en la segunda mitad del pasado siglo, dio lugar a intensos cambios sociales, económicos y culturales, que también tuvieron una repercusión importante en la educación. Hoy día existe una gran cantidad de información en formato digital en la Red que está a disposición tanto del profesorado como del alumnado, así como un número ingente de recursos que pueden ser usados para la enseñanza y aprendizaje de la geografía. Pero también son muchos los desafíos y dificultades que deben superarse para lograr una adecuada utilización y un eficaz aprovechamiento de las herramientas tecnológicas por parte de los profesionales de la educación, así como para saber transmitir su potencial al alumnado (Martín y García, 2009). Es evidente que, en la sociedad del conocimiento, carece de sentido la figura del docente cuya actuación se reduce a la de un mero transmisor de la misma información que los propios estudiantes pueden encontrar en la Red, sino que su papel debe ser el de un orientador que les ayude en la resolución de problemas (Buzo, 2015).

Pues bien, la geografía fue una de las disciplinas que mayor impacto ha experimentado con la revolución de las nuevas tecnologías y, más concretamente, con el desarrollo y difusión de Internet. En la actualidad, los usuarios (también alumnos y alumnas) ya no son sujetos receptivos que se limitan a la consulta de la información disponible, sino que participan activamente en la creación de nuevos contenidos y servicios (Capel, 2010; Buzo, 2015). A este respecto, Sui y Morrill (2004) señalan que las nuevas tecnologías, y de manera especial los ordenadores, extendieron los ojos de los geógrafos a través de la teledetección y las fotografías aéreas; sus manos, mediante la cartografía asistida por ordenador; su boca, con la propia Red y la telefonía; y sus mentes, con la ayuda del procesado de datos y la inteligencia artificial.

El profesorado de geografía ve en el uso de las nuevas tecnologías, en particular aquellas que están orientadas a la educación, una herramienta didáctica necesaria, pero con ciertas dificultades en su utilización (Buzo, 2015). Hay que tener en cuenta que cada día existe más información y aplicaciones en la Red, lo que requiere mucho tiempo y esfuerzo para ponerse al día en todas las novedades que aparecen en cada materia (Martín y García, 2009). Por otro lado, la amplia difusión de las conexiones a la Red mediante alta velocidad, gracias a los teléfonos móviles de última generación, no se ha visto seguida de un incremento notable en el uso de estos recursos tecnológicos. En la práctica, la utilización del ordenador en el aula limita su función a la de un dispositivo meramente expositivo, a modo de proyector, para el uso de presentaciones o para buscar información; es decir, se emplea como herramienta docente, de igual forma que en la década de los años ochenta del pasado siglo y sin modificar el modelo didáctico (Martín y García, 2009).

Frente a esta realidad, lo que planteamos, siguiendo a De Miguel (2016), es que la utilización de la cartografía digital y los sistemas de información geográfica (SIG) deben representar esa parte necesaria de renovación curricular y metodológica de la didáctica de la geografía y de las ciencias sociales. Los SIG suponen una herramienta que posibilita la realización de análisis espaciales muy completos, al permitir el manejo de mucha información relativa a un punto concreto de la superficie terrestre (De Lázaro y González, 2005). Del mismo modo, facilitan la adquisición, el almacenamiento, la recuperación, la gestión y la interpretación de la información espacialmente georreferenciada con datos que pueden proceder tanto de fuentes tradicionales (estadísticas, mapas, archivos etc.) como de satélites, sensores remotos o fotointerpretación (Capel, 2010). 
Es evidente que los SIG y, en especial, los Web-SIG constituyen un recurso didáctico mucho más atractivo y dinámico que el libro de texto, ya que el alumnado puede seleccionar y combinar distintas capas de información geográfica, lo que le ayuda a comprender la compleja interacción de los factores físicos y humanos en la organización del territorio, así como a desarrollar un pensamiento crítico y creativo (De Miguel, 2016). El traslado de los SIG a la Red permite que esta tecnología sea utilizada y aprovechada por cualquier usuario, facilitando a la vez su inclusión en el proceso de enseñanza-aprendizaje (De Lázaro y González, 2005).

Además de los SIG y los SIG-Web existen otras aplicaciones, como es el caso de los visores web, los atlas en línea, las aplicaciones desarrolladas por Google (Google Earth, Google Maps, Google Street View), así como otros recursos digitales que pueden utilizarse en las aulas para la enseñanza y aprendizaje de la geografía y de las ciencias sociales. Cada día es más factible utilizar la información procedente de la Red, bien sea a través de la descarga directa o mediante la utilización de los servicios que ofrecen los estándares del Open Geospatial Consortium (OGC), como Web Map Service (WMS) o Web Feature Service (WFS), que permiten visualizar y descargar datos para trabajar con ellos en el ordenador personal (González y Lázaro, 2011). Todas estas herramientas están cambiando los contenidos y los métodos de enseñar y aprender geografía en las aulas y, además, llevan asociada una reflexión sobre qué y cómo enseñar geografía a niños y jóvenes (De Miguel, 2016).

Una de las fuentes de datos más prolíficas es la Infraestructura de Datos Espaciales de España (IDEE), cuyo fin es integrar, a través de la Red y con unas normas de interoperabilidad, los datos y servicios de tipo geográfico que se producen en España a nivel estatal, autonómico y local. La IDDE forma parte, a su vez, de la Infraestructura de Datos Espaciales Europea, que establece una infraestructura de información espacial en la Unión Europea basada en las infraestructuras de información geográfica creadas por los Estados miembros. Además, cada Comunidad Autónoma tiene una IDE propia y, desde ellas, los usuarios tienen la posibilidad de buscar y descargar ficheros de información geográfica, así como herramientas para visualizar la cartografía e imágenes de diferentes fuentes. Constituye, por tanto, una herramienta muy valiosa para trabajar en las aulas e impulsar el aprendizaje por descubrimiento, desarrollar proyectos de análisis espacial y, en definitiva, contribuir al proceso de alfabetización cartográfica para ayudar a los estudiantes a entender mejor el mundo en el que viven (Armas, Rodríguez y Macía, 2017).

\section{ConClusión}

Está claro que una geografía descriptiva basada en una relación de países, capitales, ríos, montañas, cifras demográficas y recursos productivos, tal como sigue proponiéndose en buena medida en los currículos escolares y en los libros de texto, es una disciplina inútil, por no decir absurda. Frente a este enfoque, el propósito de la educación geográfica debe dirigirse a "razonar geográficamente, pensar el espacio" (Clary, 1992, p. 33); se trata de que el conjunto de la ciudadanía, y no solo la población escolar, "piense geográficamente”, es decir, use el razonamiento espacial para desenvolverse en el mundo y para mejorar su relación con el territorio (Gersmehl, 2014). En definitiva, si queremos dejar de hablar de una disciplina académica irrelevante, debemos apostar por una geografía escolar que, partiendo de una problematización de los contenidos, se oriente nada más y nada menos que a "educar geográficamente” (Souto y Ramírez, 1996, p. 18) a la ciudadanía.

Por otro lado, desde un planteamiento crítico resulta determinante tener muy presente que la cartografía nunca es inocente. Por el contrario, los mapas son artefactos cargados de poder, sea este de naturaleza política, religiosa, militar o de cualquier otro signo. Por eso, algunos documentos cartográficos desvelan información, mientras que otros tratan precisamente de esconderla; a veces proporcionan datos, pero en otras ocasiones los ocultan. La intencionalidad del lenguaje cartográfico se convierte así en un punto de interés prioritario, ya que cada mapa pretende dar 
respuesta a un asunto concreto, lo que representa una determinada selección y presentación de la información para lograr una visualización eficaz de la cuestión que lo ha justificado. Y no solo eso, sino que la utilización de uno u otro tipo de mapas tiene una incidencia directa en la elección de los temas que vamos a considerar objeto de enseñanza.

Parece claro, pues, que la formación del profesorado con relación a la alfabetización cartográfica debe ser coherente con la misión que se le atribuya a la geografía como disciplina. Si partimos de la convicción de que la geografía escolar debe servir para ayudar a los estudiantes a que comprendan críticamente el mundo en el que viven, con la finalidad última de que sean capaces de transformarlo, entonces tenemos que trasladar al profesorado la idea de que los mapas son instrumentos que facilitan ese análisis reflexivo y comprometido en la búsqueda de soluciones a los problemas derivados de la interacción entre espacio y grupos sociales. Por consiguiente, el mapa debe ser un recurso didáctico que permita formular hipótesis explicativas y juicios críticos, convirtiéndose así en "un buen instrumento para desvelar problemas del análisis sociopolítico de la realidad” (Calaf, Suárez y Menéndez, 1997, p. 155) y para ayudar a construir un conocimiento valorativo.

Desde esta perspectiva, es preciso que los estudiantes diferencien entre la realidad espacial y su representación simbólica a través del lenguaje cartográfico, y que el profesorado conozca las secuencias y dificultades en el aprendizaje de las habilidades cartográficas (Souto, 1999). Centrándonos en las responsabilidades docentes a este respecto, no es discutible la necesidad de una sólida formación del profesorado en relación con las competencias necesarias para conducir el proceso de enseñanza y aprendizaje del lenguaje cartográfico en la escuela. Saber "leer” un mapa es un conocimiento necesario, pero en ningún modo es automático ni espontáneo, sino que tiene que ser aprendido (Thrower, 2002). De la misma forma que existen métodos y enfoques para la adquisición del lenguaje oral y escrito, la alfabetización cartográfica exige también el empleo de una metodología específica que permita el desarrollo del pensamiento espacial, y los docentes deben estar en posesión de las competencias profesionales necesarias para llevar a cabo esta tarea.

\section{Referencias}

Armas Quintá, F. X., Rodríguez Lestegás, F. y Macía Arce, X. C. (2017). Os SIX como recurso didáctico para o ensino e aprendizaxe da xeografía na educación secundaria. Revista Galega de Educación, 69, 28-31.

Audigier, F. (1994). La didactique de la Géographie. En P. Desplanques (Coord.), La Géographie en collège et en lycée (pp. 102-127). París: Hachette.

Bailey, P. (1981). Didáctica de la Geografía. Madrid: Cincel-Kapelusz.

Benejam, P. y Comes, P. (1994). ¿Nuevas coordenadas para la enseñanza de la Geografía? Las implicaciones del constructivismo en la enseñanza-aprendizaje de las habilidades cartográficas. Íber. Didáctica de las Ciencias Sociales, Geografía e Historia, 1, 106-116.

Brotton, J. (2014). Historia del mundo en 12 mapas. Barcelona: Debate.

Buzo Sánchez, I. (2015). Posibilidades y límites de las TIC en la enseñanza de la geografía. Ar@cne: Revista Electrónica de Recursos en Internet sobre Geografía y Ciencias Sociales, 195. Recuperado de http://www.ub.edu/geocrit/aracne/aracne-195.pdf

Calaf, R., Suárez, M. A. y Menéndez, R. (1997). Aprender a enseñar geografía. Barcelona: OikosTau.

Capel, H. (2010). Geografía en red a comienzos del tercer milenio: para una ciencia solidaria y en colaboración. Scripta Nova: Revista Electrónica de Geografía y Ciencias Sociales, XIV(313). Recuperado de http://www.ub.edu/geocrit/sn/sn-313.htm

Catalá Romero, R. (2016). Explicando el mundo en el que vivimos con mapas: Propuesta de aprendizaje cartográfico. GeoGraphos, 7(89), 171-206. DOI: 10.14198/GEOGRA2016.7.89 
Cavalcanti, L. S. (1998). Geografia, escola e construção de conhecimentos. Campinas: SP. Papirus Editora.

Cavalcanti, L. S. (2005). Ensino de Geografia e diversidade: construção de conhecimentos geográficos escolares e atribuição de significados pelos diversos sujeitos do processo de ensino. En S. Castellar (Org.), Educação geográfica: teorías e práticas docentes (pp. 66-78). São Paulo: Contexto.

Cavalcanti, L. S. (2012). La geografía escolar en Brasil y desafíos para la práctica de la enseñanza. Geoenseñanza, 17(1), 23-38.

Clary, M. (1992). Aprender a situar, situar para aprender. Boletín de Didáctica de las Ciencias Sociales, 5, 31-43.

De Lázaro Torres, M. L. y González González, M. J. (2005). La utilidad de los sistemas de información geográfica para la enseñanza de la geografía. Didáctica Geográfica 7, 105-122.

De Miguel González, R, (2013). Geoinformación e innovación en la enseñanza-aprendizaje de la geografía: un reto pendiente en los libros de texto de secundaria. Didáctica de las Ciencias Experimentales y Sociales, 27, 67-90.

De Miguel González, R. (2016). Espacio digital y educación geográfica: el Atlas Digital Escolar. En R. López Facal (Ed.), VII Simposio de Didáctica de las Ciencias Sociales en el ámbito Iberoamericano. Ciencias sociales, educación y futuro. Investigaciones en didáctica de las ciencias sociales (pp. 1148-1160). Santiago de Compostela: Red14-Universidad de Santiago de Compostela.

Duarte, R. G. (2017). A cartografia escolar e o pensamento (geo)espacial: alicerces da educação geográfica. En V. O. Roque Ascenção, R. C. Valadão, R. Soares del Gaudio y C. J. O. Souza (Orgs.), Conhecimentos da Geografia: Percursos de Formação Docente e Práticas na Educação Básica (pp. 28-52). Belo Horizonte: Universidade Federal de Minas Gerais.

Eisner, E. W. (1979). The Educational Imagination: on the Design and Evaluation of School Programs. Nueva York: Macmillan Publishing.

Fontanabona, J. (Dir.) (2000). Cartes et modèles graphiques: analyses de pratiques en classe de géographie. París: I.N.R.P.

Gersmehl, P. (2014). Teaching geography (Third edition). Nueva York: Guilford Press.

González González, M. J. y Lázaro Torres, M. L. (2011). La geoinformación y su importancia para las tecnologías de la información geográfica. Ar@cne: Revista Electrónica de Recursos en Internet sobre Geografía y Ciencias Sociales, $148 . \quad$ Recuperado de http://www.ub.edu/geocrit/aracne/aracne-148.htm

Grataloup, C. y Fumey, G. (Dirs.) (2016). Atlas Global. Madrid: Cátedra.

Hartshorne, R. (1939). The Nature of Geography: A Critical Survey of Current Thought in the Light of the Past. Annals (Association of American Geographers), 29, 171-645.

Jackson, P. W. (1968). Life in Classrooms. Nueva York: Holt, Rinehart \& Winston.

Jerez, O. (2006). El lenguaje cartográfico como instrumento para la enseñanza de una geografía crítica y para la educación ambiental. En M. J. Marrón, L. Sánchez y O. Jerez (Coords.), Cultura geográfica y educación ciudadana (pp. 483-501). Cuenca: Ediciones de la Universidad de Castilla-La Mancha.

Lacoste, Y. (1977). La geografía: un arma para la guerra. Barcelona: Anagrama.

Luque, R. (2011). El uso de la cartografía y la imagen digital como recurso didáctico en la enseñanza secundaria. Algunas precisiones en torno a Google Earth. Boletín de la Asociación de Geógrafos Españoles, 55, 183-210.

Martín Gómez, C. y García Pérez, F. F. (2009). Algunos recursos en Internet para mejorar la enseñanza de la geografía. Ar@cne: Revista Electrónica de Recursos en Internet sobre Geografía y Ciencias Sociales, 118. Recuperado de http://www.ub.edu/geocrit/aracne/aracne$\underline{118 . h t m}$ 
National Research Council (2006). Learning to Think Spatially: GIS as a Support System in the K12 Curriculum. Washington: The National Academies Press.

Nieto, G. y Siegmund, A. (2019). Examining the educative practice with Geographic Information Systems through the teachers’ perspective. Didáctica Geográfica, 20, 153-171.

Peters, A. (1992). La nueva cartografía. Barcelona: Vicens Vives.

Richter, D. y Cavallini, G. M. (2019). El lenguaje cartográfico en los libros de texto de geografía de secundaria en Brasil. Didáctica Geográfica, 20, 193-212.

Sinton, D. S., Bednarz, S., Gersmehl, P., Kolvoord, R. y Uttal, D. (2013). The People's Guide to Spatial Thinking. Washington: National Council for Geographic Education.

Souto, X. M. (1999). Didáctica de la geografía: problemas sociales y conocimiento del medio. Barcelona: Ediciones del Serbal.

Souto, X. M. y Ramírez, S. (1996). Enseñar Geografía o educar geográficamente a las personas. Íber. Didáctica de las Ciencias Sociales, Geografía e Historia, 9, 15-26.

Sui, D. y Morrill, R. (2004). Computers and geography: from automated geography to digital earth. En S. D. Brunn, S. L. Cutter y J. W. Harrington Jr. (Eds.), Geography and Technology (pp. 81-108). Amsterdam: Kluwer Academic Publishers.

Thrower, N. J. W. (2002). Mapas y civilización: historia de la cartografía en su contexto cultural y social. Barcelona: Ediciones del Serbal.

Zwartjes, L. y de Lázaro, M. L. (2019). Geospatial Thinking Learning Lines in Secondary Education: The GI Learner Project. En R. de Miguel, K. Donert y K. Koutsopoulos (Eds.), Geospatial Technologies in Geography Education. Key Challenges in Geography (EUROGEO Book Series) (pp. 41-61). Cham: Springer. DOI: 10.1007/978-3-030-17783-6_3

\section{CÓMO CITAR ESTE ARTÍ́CULO}

Rodríguez Lestegás, F, Macía Arce, X. C. y Armas Quintá, F. X. (2021). Alfabetización cartográfica: un desafío para la formación del profesorado. Didáctica de las ciencias experimentales y sociales, 40, 67-80. DOI: 10.7203/DCES.40.18054 